\title{
Chlorinated and brominated polycyclic aromatic hydrocarbons on the Tibetan Plateau
}

GUORUI LIU, MINGHUI ZHENG, RONG JIN, LILI YANG, CUI LI, XIAOYUN LIU

State Key Laboratory of Environmental Chemistry and Ecotoxicology, Research Center for Eco-Environmental Sciences, Chinese Academy of Sciences, Beijing 100085, China

Chlorinated and brominated polycyclic aromatic hydrocarbons (Cl/Br-PAHs; XPAHs $\geq 3$ rings) are highly toxic halogenated derivatives of polycyclic aromatic hydrocarbons (PAHs), which have similar toxicity to that of dioxins. The Tibetan Plateau is the largest elevated plateau in the world. It lies in the southwest of China and is one of the coldest and most pristine regions in the world. The large vertical temperatures and precipitation gradients may cause enrichment of semi-volatile and long-lived pollutants in high altitudes. Therefore, the Tibetan Plateau is pivotal for the transport and regional to global distribution of persistent organic pollutants. Air, soil and vegetation are important matrices that could be used for clarifying the abundance, congener patterns and environmental behaviors of POPs in remote areas. In this study, air, soils, lichens, and mosses were collected in Medog County and in the Shergyla Mountains, areas located in the southeast of the Tibetan Plateau. Seventeen PAHs, nineteen ClPAHs and nineteen BrPAHs were targeted and detected in these samples for the first time.

The alpine behaviors of XPAHs were clarified by using lichens as the bio-indicator of XPAHs in air. The enrichment of photochemically long-lived XPAHs in the high mountain environment is influenced by the physicochemical properties of XPAHs. Anthropogenic emissions and photochemical formation along long-range transport to the remote environment contribute $47 \%$ and $28 \%$, respectively, to chlorinated PAHs abundant on the Tibetan Plateau. 